\title{
A NEW DECOMPOSITION FOR SQUARE MATRICES*
}

\author{
JULIO BENíTEZ
}

\begin{abstract}
A new decomposition is derived for any complex square matrix. This decomposition is based on the canonical angles between the column space of this matrix and the column space of its conjugate transpose. Some applications of this factorization are given; in particular some matrix partial orderings and the relationship between the canonical angles and various classes of matrices are studied.
\end{abstract}

Key words. Decomposition of matrices, EP matrices, Canonical angles, Matrix partial ordering.

AMS subject classifications. 15A23, 15A57.

1. Introduction. Let $\mathbb{C}_{m, n}$ be the set of $m \times n$ complex matrices, and let $A^{*}$, $\mathcal{R}(A), \mathcal{N}(A)$, and $\operatorname{rank}(A)$ denote the conjugate transpose, column space, null space, and rank, respectively, of $A \in \mathbb{C}_{m, n}$. For a nonsingular $A \in \mathbb{C}_{n, n}$, we shall denote $A^{-*}=\left(A^{-1}\right)^{*}=\left(A^{*}\right)^{-1}$. Furthermore, let $A^{\dagger}$ stand for the Moore-Penrose inverse of $A$, i.e., for the unique matrix satisfying the equations

$$
A A^{\dagger} A=A, \quad A^{\dagger} A A^{\dagger}=A^{\dagger}, \quad A A^{\dagger}=\left(A A^{\dagger}\right)^{*}, \quad A^{\dagger} A=\left(A^{\dagger} A\right)^{*} .
$$

Given a matrix $A \in \mathbb{C}_{n, n}$, it can be proved that the set $\left\{X \in \mathbb{C}_{n, n}: A X A=\right.$ $A, X A X=X, A X=X A\}$ is empty or a singleton. When it is a singleton, it is customary to denote by $A^{\#}$ its unique element, called the group inverse of $A$. Furthermore, $I_{n}$ means the identity matrix of order $n$. We shall denote by $0_{n, m}$ the zero matrix in $\mathbb{C}_{n, m}$, and when there is no danger of confusion, we will simply write 0 . In addition, $\mathbf{1}_{n}$ and $\mathbf{0}_{n}$ will denote the $n \times 1$ column vectors all of whose components are 1 and 0 , respectively.

Hartwig and Spindelböck arrived at the following result, given in [14] as Corollary 6 .

Theorem 1.1. Let $A \in \mathbb{C}_{n, n}$ and $r=\operatorname{rank}(A)$. There exist a unitary $U \in \mathbb{C}_{n, n}$, $\Sigma=\sigma_{1} I_{r_{1}} \oplus \cdots \oplus \sigma_{t} I_{r_{t}}, r_{1}+\cdots+r_{t}=r, \sigma_{1}>\cdots>\sigma_{t}>0, L \in \mathbb{C}_{r, n-r}, K \in \mathbb{C}_{r, r}$

\footnotetext{
${ }^{*}$ Received by the editors November 17, 2009. Accepted for publication April 2, 2010. Handling Editor: Roger A. Horn.

$\dagger$ Departamento de Matemática Aplicada, Instituto de Matemática Multidisciplinar, Universidad Politécnica de Valencia, Camino de Vera s/n, 46022, Valencia, Spain. (jbenitez@mat.upv.es).
} 
such that

$$
A=U\left[\begin{array}{cc}
\Sigma K & \Sigma L \\
0 & 0
\end{array}\right] U^{*}
$$

and

$$
K K^{*}+L L^{*}=I_{r} .
$$

Straightforward computations show that if $A$ is written as in (1.1), then

$$
A^{\dagger}=U\left[\begin{array}{cc}
K^{*} \Sigma^{-1} & 0 \\
L^{*} \Sigma^{-1} & 0
\end{array}\right] U^{*}
$$

The usefulness of the representation provided in Theorem 1.1 to explore various classes of matrices, was demonstrated in $[2,3,24]$.

In the sequel, $\|K\|$ with $K \in \mathbb{C}_{m, n}$ will be the matrix norm induced by the Euclidean vector norm (known as the spectral norm); see [18, pp 270, 281]. One has that if $U, V$ are unitary, then $\left\|U A V^{*}\right\|=\|A\|$ for any matrix $A$ such that the product $U A V^{*}$ is meaningful $([18, \mathrm{p} 283])$. Also it will be needed that $\|A\|^{2}=\left\|A^{*}\right\|^{2}=$ $\left\|A^{*} A\right\|=\left\|A A^{*}\right\|$ holds for any complex matrix.

The canonical angles (also called principal angles) between two subspaces provide the best available characterization of the relative position of two given subspaces. This concept allows us to characterize or measure, in a natural way, how two subspaces differ, which is the main connection with perturbation theory. In $[9,20,22]$ we can find how these angles were discovered and rediscovered again several times. Computation of canonical angles between subspaces is important in many applications including statistics [8, 15], information retrieval [16], and analysis of algorithms [23]. There are many equivalent definitions of the canonical angles (see [11]). But, for our purposes, the most convenient is the following:

Let $\mathcal{X}$ and $\mathcal{Y}$ be two nontrivial subspaces of $\mathbb{C}^{n}$ and $r=\min \{\operatorname{dim} \mathcal{X}, \operatorname{dim} \mathcal{Y}\}$. We define the canonical angles $\theta_{1}, \ldots, \theta_{r} \in[0, \pi / 2]$ between $\mathcal{X}$ and $\mathcal{Y}$ by

$$
\cos \theta_{i}=\sigma_{i}\left(P_{\mathcal{X}} P_{\mathcal{Y}}\right), \quad i=1, \ldots, r
$$

where the nonnegative real numbers $\sigma_{1}\left(P_{\mathcal{X}} P_{\mathcal{Y}}\right), \ldots, \sigma_{r}\left(P_{\mathcal{X}} P_{\mathcal{Y}}\right)$ are the singular values of $P_{\mathcal{X}} P_{\mathcal{Y}}$. Here, $P_{\mathcal{S}}$ stands for the orthogonal projector onto the subspace $\mathcal{S} \subset \mathbb{C}^{n}$. We will have in mind the possibility that one canonical angle is repeated.

See also $[11,12,21,25]$ for the study of this definition and [5] for the simultaneous study of two orthogonal projectors by means of the canonical angles between the column spaces of these orthogonal projectors. 
2. Main result. The following theorem is the main result of the paper.

Theorem 2.1. Let $A \in \mathbb{C}_{n, n}$, let $r=\operatorname{rank}(A)$, and let $\theta_{1}, \ldots, \theta_{p}$ be the canonical angles between $\mathcal{R}(A)$ and $\mathcal{R}\left(A^{*}\right)$ belonging to $] 0, \pi / 2[$. Denote by $x$ and $y$ the multiplicities of the angles 0 and $\pi / 2$ as a canonical angle between $\mathcal{R}(A)$ and $\mathcal{R}\left(A^{*}\right)$, respectively. There exists a unitary matrix $Y \in \mathbb{C}_{n, n}$ such that

$$
A=Y\left[\begin{array}{cc}
M C & M S \\
0 & 0
\end{array}\right] Y^{*},
$$

where $M \in \mathbb{C}_{r, r}$ is nonsingular,

$$
\begin{gathered}
C=\operatorname{diag}\left(\mathbf{0}_{y}, \cos \theta_{1}, \ldots, \cos \theta_{p}, \mathbf{1}_{x}\right), \\
S=\left[\begin{array}{cc}
\operatorname{diag}\left(\sin \theta_{1}, \ldots, \sin \theta_{p}, \mathbf{1}_{y}\right) & 0_{p+y, n-(r+p+y)} \\
0_{x, p+y} & 0_{x, n-(r+p+y)}
\end{array}\right],
\end{gathered}
$$

and $r=y+p+x$. Furthermore, $x$ and $y+n-r$ are the multiplicities of the singular values 1 and 0 in $P_{\mathcal{R}(A)} P_{\mathcal{R}\left(A^{*}\right)}$, respectively.

Proof. Let us represent $A$ as in (1.1). From this representation and (1.3) we have

$$
A^{\dagger} A=U\left[\begin{array}{cc}
K^{*} K & K^{*} L \\
L^{*} K & L^{*} L
\end{array}\right] U^{*}
$$

Now, let us observe $\|K\|^{2}=\left\|K^{*} K\right\| \leq\left\|A A^{\dagger}\right\|=1$, since the norm of a submatrix cannot be greater than the norm of the matrix [1, Lemma 2] and the spectral norm of any orthogonal projector is 1 . Let $K=V C W^{*}$ be the singular value decomposition of $K$, where $V, W \in \mathbb{C}_{r, r}$ are unitary and $C \in \mathbb{C}_{r, r}$ is diagonal with nonnegative real numbers on its diagonal. Since $\|C\|=\|K\| \leq 1$, we can write

$$
C=\operatorname{diag}\left(\mathbf{0}_{y}, \cos \theta_{1}, \ldots, \cos \theta_{p}, \mathbf{1}_{x}\right),
$$

where $y, p, x \in \mathbb{N} \cup\{0\}$ satisfy $y+p+x=r$, and $\left.\theta_{1}, \ldots, \theta_{p} \in\right] 0, \pi / 2[$.

Let us define

$$
\widehat{C}=\operatorname{diag}\left(\mathbf{0}_{y}, \cos \theta_{1}, \ldots, \cos \theta_{p}\right) \quad \text { and } \quad \widehat{S}=\operatorname{diag}\left(\mathbf{1}_{y}, \sin \theta_{1}, \ldots, \sin \theta_{p}\right) .
$$

Obviously, $\widehat{S}$ is nonsingular and

$$
\widehat{C}^{2}+\widehat{S}^{2}=I_{y+p} .
$$

Now, let us partition $V$ and $W$ as follows:

(2.6) $V=\left[\begin{array}{ll}V_{1} & V_{2}\end{array}\right], \quad W=\left[\begin{array}{ll}W_{1} & W_{2}\end{array}\right], \quad V_{1}, W_{1} \in \mathbb{C}_{r, y+p}, V_{2}, W_{2} \in \mathbb{C}_{r, x}$. 
Since $V$ is unitary,

$$
\left[\begin{array}{cc}
I_{y+p} & 0 \\
0 & I_{x}
\end{array}\right]=I_{r}=V^{*} V=\left[\begin{array}{c}
V_{1}^{*} \\
V_{2}^{*}
\end{array}\right]\left[\begin{array}{ll}
V_{1} & V_{2}
\end{array}\right]=\left[\begin{array}{ll}
V_{1}^{*} V_{1} & V_{1}^{*} V_{2} \\
V_{2}^{*} V_{1} & V_{2}^{*} V_{2}
\end{array}\right],
$$

hence

$$
I_{y+p}=V_{1}^{*} V_{1}, \quad 0=V_{1}^{*} V_{2}, \quad 0=V_{2}^{*} V_{1}, \quad I_{x}=V_{2}^{*} V_{2} .
$$

Using again that $V$ is unitary we get

$$
I_{r}=V_{1} V_{1}^{*}=\left[\begin{array}{ll}
V_{1} & V_{2}
\end{array}\right]\left[\begin{array}{c}
V_{1}^{*} \\
V_{2}^{*}
\end{array}\right]=V_{1} V_{1}^{*}+V_{2} V_{2}^{*} .
$$

Similarly, we get

$$
I_{y+p}=W_{1}^{*} W_{1}, \quad 0=W_{1}^{*} W_{2}, \quad 0=W_{2}^{*} W_{1}, \quad I_{x}=W_{2}^{*} W_{2}
$$

and $I_{r}=W_{1} W_{1}^{*}+W_{2} W_{2}^{*}$.

From the singular value decomposition of $K,(2.4)$, and (2.6) we get

$$
K=V C W^{*}=\left[\begin{array}{ll}
V_{1} & V_{2}
\end{array}\right]\left[\begin{array}{cc}
\widehat{C} & 0 \\
0 & I_{x}
\end{array}\right]\left[\begin{array}{l}
W_{1}^{*} \\
W_{2}^{*}
\end{array}\right]=V_{1} \widehat{C} W_{1}^{*}+V_{2} W_{2}^{*} .
$$

Now, let us prove

$$
L L^{*}=V_{1} \widehat{S}^{2} V_{1}^{*}
$$

In fact, the combination of (1.2), (2.5), (2.8), (2.9), and (2.10) leads to

$$
\begin{aligned}
L L^{*} & =I_{r}-K K^{*} \\
& =I_{r}-\left(V_{1} \widehat{C} W_{1}^{*}+V_{2} W_{2}^{*}\right)\left(V_{1} \widehat{C} W_{1}^{*}+V_{2} W_{2}^{*}\right)^{*} \\
& =I_{r}-\left(V_{1} \widehat{C} W_{1}^{*}+V_{2} W_{2}^{*}\right)\left(W_{1} \widehat{C} V_{1}^{*}+W_{2} V_{2}^{*}\right) \\
& =I_{r}-V_{1} \widehat{C}^{2} V_{1}^{*}-V_{2} V_{2}^{*} \\
& =V_{1} V_{1}^{*}-V_{1} \widehat{C}^{2} V_{1}^{*} \\
& =V_{1}\left(I_{p+y}-\widehat{C}^{2}\right) V_{1}^{*} \\
& =V_{1} \widehat{S}^{2} V_{1}^{*} .
\end{aligned}
$$

Now, we have from (2.7) and (2.11)

$$
V_{1}^{*} L L^{*} V_{1}=V_{1}^{*} V_{1} \widehat{S}^{2} V_{1}^{*} V_{1}=\widehat{S}^{2} .
$$

Let us define

$$
X_{1}=L^{*} V_{1} \widehat{S}^{-1}, \quad X_{1} \in \mathbb{C}_{n-r, y+p}
$$


Apply (2.12) and the definition of $X_{1}$ to obtain $X_{1}^{*} X_{1}=\widehat{S}^{-1} V_{1}^{*} L L^{*} V_{1} \widehat{S}^{-1}=I_{y+p}$, therefore, the columns of $X_{1}$ are orthonormal. As $X_{1}$ has $y+p$ orthonormal columns belonging to $\mathbb{C}^{n-r}$, we have $y+p \leq n-r$. Let us define $t=(n-r)-(y+p) \in \mathbb{N} \cup\{0\}$. We can find $X_{2} \in \mathbb{C}_{n-r, t}$ such that $X=\left[X_{1} X_{2}\right]$ is unitary. Finally, let us define

$$
S=\left[\begin{array}{cc}
\widehat{S} & 0_{y+p, t} \\
0_{x, y+p} & 0_{x, t}
\end{array}\right] \in \mathbb{C}_{r, n-r} .
$$

From $\widehat{C}^{2}+\widehat{S}^{2}=I_{y+p}$ and $\widehat{C} \widehat{S}=\widehat{S} \widehat{C}$ we get

$$
C^{2}+S S^{*}=I_{r}, \quad\left(\widehat{C} \oplus I_{t}\right)^{2}+S^{*} S=I_{n-r}, \quad C S=S\left(\widehat{C} \oplus I_{t}\right) .
$$

Since $X$ is unitary we get

$$
X_{1}^{*} X_{2}=0 .
$$

From (2.7) and (2.11) we get $\left(L^{*} V_{2}\right)^{*}\left(L^{*} V_{2}\right)=V_{2}^{*} L L^{*} V_{2}=V_{2}^{*} V_{1} \widehat{S}^{2} V_{1}^{*} V_{2}=0$, hence

$$
L^{*} V_{2}=0
$$

Taking into account (2.13) and (2.17),

$$
L^{*} V=L^{*}\left[\begin{array}{ll}
V_{1} & V_{2}
\end{array}\right]=\left[\begin{array}{ll}
L^{*} V_{1} & L^{*} V_{2}
\end{array}\right]=\left[\begin{array}{ll}
X_{1} \widehat{S} & 0
\end{array}\right] .
$$

From which, and having in mind that $V$ is unitary and $\widehat{S}$ is Hermitian, we get

$$
L=V\left[\begin{array}{c}
\widehat{S} X_{1}^{*} \\
0
\end{array}\right]=\left[\begin{array}{ll}
V_{1} & V_{2}
\end{array}\right]\left[\begin{array}{c}
\widehat{S} X_{1}^{*} \\
0
\end{array}\right]=V_{1} \widehat{S} X_{1}^{*} .
$$

By using (2.16) and postmultiplying (2.18) by $X_{2}$ we get

$$
L X_{2}=0 .
$$

From (2.7), (2.11), (2.13), and (2.19) we obtain

$$
\begin{aligned}
& \text { (2.20) } L X= \\
& =L\left[\begin{array}{ll}
X_{1} & X_{2}
\end{array}\right]=\left[\begin{array}{ll}
L X_{1} & L X_{2}
\end{array}\right]=\left[\begin{array}{ll}
L L^{*} V_{1} \widehat{S}^{-1} & 0
\end{array}\right]=\left[\begin{array}{ll}
V_{1} \widehat{S} & 0
\end{array}\right] .
\end{aligned}
$$

On the other hand, we have

$$
V S=\left[\begin{array}{ll}
V_{1} & V_{2}
\end{array}\right]\left[\begin{array}{cc}
\widehat{S} & 0 \\
0 & 0
\end{array}\right]=\left[\begin{array}{ll}
V_{1} \widehat{S} & 0
\end{array}\right] .
$$


Relations (2.20) and (2.21) prove $L=V S X^{*}$. Moreover, observe

$$
\begin{aligned}
A & =U\left[\begin{array}{cc}
\Sigma K & \Sigma L \\
0 & 0
\end{array}\right] U^{*} \\
& =U\left[\begin{array}{cc}
\Sigma V C W^{*} & \Sigma V S X^{*} \\
0 & 0
\end{array}\right] U^{*} \\
& =U\left[\begin{array}{cc}
\Sigma V C & \Sigma V S \\
0 & 0
\end{array}\right]\left[\begin{array}{cc}
W^{*} & 0 \\
0 & X^{*}
\end{array}\right] U^{*} \\
& =U\left[\begin{array}{cc}
W & 0 \\
0 & X
\end{array}\right]\left[\begin{array}{cc}
W^{*} \Sigma V C & W^{*} \Sigma V S \\
0 & 0
\end{array}\right]\left[\begin{array}{cc}
W^{*} & 0 \\
0 & X^{*}
\end{array}\right] U^{*} .
\end{aligned}
$$

Thus, if we denote $Y=U(W \oplus X)$ and $M=W^{*} \Sigma V$, we have

$$
A=Y\left[\begin{array}{cc}
M C & M S \\
0 & 0
\end{array}\right] Y^{*}
$$

Notice that $Y$ is unitary and $M$ is nonsingular.

It remains to prove that $\theta_{1}, \ldots, \theta_{p}$ are the canonical angles between $\mathcal{R}(A)$ and $\mathcal{R}\left(A^{*}\right)$ belonging to $] 0, \pi / 2[$, and $x$ and $y$ are the multiplicities of the singular values 0 and 1 in $P_{\mathcal{R}(A)} P_{\mathcal{R}\left(A^{*}\right)}$, respectively. To this end, we will use (1.4). It is straightforward by checking the four conditions of the Moore-Penrose inverse that if $A$ is written as in $(2.1)$, then

$$
A^{\dagger}=Y\left[\begin{array}{cc}
C M^{-1} & 0 \\
S^{*} M^{-1} & 0
\end{array}\right] Y^{*}
$$

Now, from (2.15), it is simple to verify

$$
\begin{aligned}
P_{\mathcal{R}(A)} P_{\mathcal{R}\left(A^{*}\right)} & =A A^{\dagger} A^{\dagger} A \\
& =Y\left[\begin{array}{cc}
M C & M S \\
0 & 0
\end{array}\right]\left[\begin{array}{ll}
C M^{-1} & 0 \\
S^{*} M^{-1} & 0
\end{array}\right]\left[\begin{array}{cc}
C M^{-1} & 0 \\
S^{*} M^{-1} & 0
\end{array}\right]\left[\begin{array}{cc}
M C & M S \\
0 & 0
\end{array}\right] Y^{*} \\
& =Y\left[\begin{array}{cc}
I_{r} & 0 \\
0 & 0
\end{array}\right]\left[\begin{array}{cc}
C^{2} & C S \\
S^{*} C & S^{*} S
\end{array}\right] Y^{*} \\
(2.23) & =Y\left[\begin{array}{cc}
C^{2} & C S \\
0 & 0
\end{array}\right] Y^{*} .
\end{aligned}
$$

Next, we are going to find the singular value decomposition of $P_{\mathcal{R}(A)} P_{\mathcal{R}\left(A^{*}\right)}$. Let us remark that from (2.15) we get that the matrix

$$
T=\left[\begin{array}{cc}
C & S \\
-S^{*} & \widehat{C} \oplus I_{t}
\end{array}\right] \in \mathbb{C}_{n, n}
$$


is unitary. Hence, the singular value decomposition of $P_{\mathcal{R}(A)} P_{\mathcal{R}\left(A^{*}\right)}$ is

$$
\begin{aligned}
P_{\mathcal{R}(A)} P_{\mathcal{R}\left(A^{*}\right)} & =Y\left(C \oplus 0_{n-r, n-r}\right)\left(T Y^{*}\right) \\
& =Y \operatorname{diag}(\underbrace{\underbrace{0, \ldots, 0}_{y}, \underbrace{\cos \theta_{1}, \ldots, \cos \theta_{p}}_{p}, \underbrace{1, \ldots, 1}_{r}}_{y} \underbrace{0, \ldots, 0}_{n-r})\left(T Y^{*}\right),
\end{aligned}
$$

since $Y$ and $T Y^{*}$ are unitary and $C \oplus 0_{n-r, n-r}$ is a diagonal matrix with real and nonnegative numbers on its diagonal. Therefore, these numbers are the singular values of $P_{\mathcal{R}(A)} P_{\mathcal{R}\left(A^{*}\right)}$. प

3. Applications. In this section some applications of the decomposition given in Theorem 2.1 are discussed. Let us remark that from (2.15), matrices $C$ and $S$ "almost" commute and behave as the ordinary trigonometric functions $x \mapsto \cos x$ and $x \mapsto \sin x$. An evident fact is that $C$ is Hermitian because $C$ is diagonal with real numbers on its diagonal. The following lemma will be used several times in the sequel.

Lemma 3.1. Assume that matrices $C$ and $S$ are defined as in Theorem 2.1 and let $q$ be an arbitrary positive integer. For $R_{1}, R_{2} \in \mathbb{C}_{r, q}$ we have

$$
C R_{1}=C R_{2} \text { and } S^{*} R_{1}=S^{*} R_{2} \quad \Longleftrightarrow \quad R_{1}=R_{2}
$$

and for $T_{1}, T_{2} \in \mathbb{C}_{q, r}$

$$
T_{1} C=T_{2} C \text { and } T_{1} S=T_{2} S \quad \Longleftrightarrow \quad T_{1}=T_{2} .
$$

Proof. Let us prove the first equivalence (the another one has a similar proof). The $\Leftarrow$ part is trivial. To prove the $\Rightarrow$ part, it is enough to premultiply $C R_{1}=C R_{2}$ by $C$ and premultiply $S^{*} R_{1}=S^{*} R_{2}$ by $S$, add the last two obtained equalities and use the first relation of (2.15).

3.1. The dimension of $\mathcal{R}(A) \cap \mathcal{R}\left(A^{*}\right)$ and $\mathcal{R}(A) \cap \mathcal{R}\left(A^{*}\right)^{\perp}$. We apply Theorem 2.1 to find the dimension of $\mathcal{R}(A) \cap \mathcal{R}\left(A^{*}\right)$ and $\mathcal{R}(A) \cap \mathcal{R}\left(A^{*}\right)^{\perp}$ in terms of the canonical angles between $\mathcal{R}(A)$ and $\mathcal{R}\left(A^{*}\right)$.

TheOREm 3.2. For any square complex matrix A, one has

(i) the dimension of $\mathcal{R}(A) \cap \mathcal{R}\left(A^{*}\right)$ is the multiplicity of the angle 0 as a canonical angle between $\mathcal{R}(A)$ and $\mathcal{R}\left(A^{*}\right)$.

(ii) the dimension of $\mathcal{R}(A) \cap \mathcal{R}\left(A^{*}\right)^{\perp}$ is the is the multiplicity of the angle $\pi / 2$ as a canonical angle between $\mathcal{R}(A)$ and $\mathcal{R}\left(A^{*}\right)$.

Proof. Let us represent $A$ as in (2.1). 
(i) First of all let us compute the dimension of $\mathcal{N}(A) \cap \mathcal{N}\left(A^{*}\right)$. Let $\mathbf{x} \in \mathcal{N}(A) \cap$ $\mathcal{N}\left(A^{*}\right)$ be represented as

$$
\mathbf{x}^{*}=\left[\mathbf{u}^{*} \mathbf{v}^{*}\right] Y^{*}, \quad \mathbf{u} \in \mathbb{C}_{r, 1}, \mathbf{v} \in \mathbb{C}_{n-r, 1} .
$$

Since $A \mathbf{x}=\mathbf{0}$ and $A^{*} \mathbf{x}=\mathbf{0}$ we get, respectively,

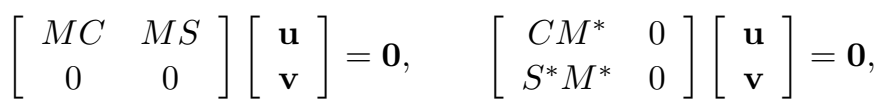

which, in view of the invertibility of $M$, reduces to

$$
C \mathbf{u}+S \mathbf{v}=\mathbf{0}, \quad C M^{*} \mathbf{u}=\mathbf{0} \text { and } S^{*} M^{*} \mathbf{u}=\mathbf{0} .
$$

From Lemma 3.1, we obtain $M^{*} \mathbf{u}=\mathbf{0}$, which implies $\mathbf{u}=\mathbf{0}$. Hence $S \mathbf{v}=\mathbf{0}$. Thus, we obtain that for $\mathbf{x}$ represented as in (3.1), one has $\mathbf{x} \in \mathcal{N}(A) \cap \mathcal{N}\left(A^{*}\right)$ if and only if $\mathbf{u}=\mathbf{0}$ and $S \mathbf{v}=\mathbf{0}$. Having in mind the representation (2.14) and the nonsingularity of $\widehat{S}$ (obtained from (2.4)), we have

$\operatorname{dim}\left[\mathcal{N}(A) \cap \mathcal{N}\left(A^{*}\right)\right]=\operatorname{dim} \mathcal{N}(S)=(n-r)-\operatorname{rank}(S)=n-r-\operatorname{rank}(\widehat{S})=n-r-(y+p)$.

Since

$$
\left[\mathcal{R}(A) \cap \mathcal{R}\left(A^{*}\right)\right]^{\perp}=\mathcal{R}(A)^{\perp}+\mathcal{R}\left(A^{*}\right)^{\perp}=\mathcal{N}\left(A^{*}\right)+\mathcal{N}(A)
$$

and

$$
\operatorname{dim}\left[\mathcal{N}(A)+\mathcal{N}\left(A^{*}\right)\right]=\operatorname{dim} \mathcal{N}(A)+\operatorname{dim} \mathcal{N}\left(A^{*}\right)-\operatorname{dim}\left[\mathcal{N}(A) \cap \mathcal{N}\left(A^{*}\right)\right],
$$

we obtain, recalling $\operatorname{dim} \mathcal{N}(A)=\mathcal{N}\left(A^{*}\right)=n-r$, that

$$
\begin{aligned}
\operatorname{dim}\left[\mathcal{R}(A) \cap \mathcal{R}\left(A^{*}\right)\right] & =n-\operatorname{dim}\left(\left[\mathcal{R}(A) \cap \mathcal{R}\left(A^{*}\right)\right]^{\perp}\right) \\
& =n-\operatorname{dim}\left(\mathcal{N}(A)+\mathcal{N}\left(A^{*}\right)\right) \\
& =n-[2(n-r)-(n-r-y-p)] \\
& =r-y-p \\
& =x .
\end{aligned}
$$

(ii) Let us recall $\mathcal{R}\left(A^{*}\right)^{\perp}=\mathcal{N}(A)$. We will prove

$$
\mathcal{R}(A) \cap \mathcal{N}(A)=\left\{Y\left[\begin{array}{l}
\mathbf{u} \\
\mathbf{0}
\end{array}\right]: \mathbf{u} \in \mathbb{C}_{r, 1}, C \mathbf{u}=\mathbf{0}\right\}
$$

To this end, let $\mathbf{x} \in \mathcal{R}(A) \cap \mathcal{N}(A)$ be represented as $\mathbf{x}^{*}=\left[\mathbf{u}^{*} \mathbf{v}^{*}\right] Y^{*}$, where $\mathbf{u} \in \mathbb{C}_{r, 1}$ and $\mathbf{v} \in \mathbb{C}_{n-r, 1}$. Since $\mathbf{x} \in \mathcal{R}(A)$ and $A A^{\dagger}$ is the orthogonal projector onto $\mathcal{R}(A)$, we 
have $A A^{\dagger} \mathbf{x}=\mathbf{x}$. Therefore using the representations (2.1) and (2.22) we easily get $\mathbf{v}=\mathbf{0}$. Since $\mathbf{x} \in \mathcal{N}(A)$, using again representation (2.1) and the nonsingularity of $M$ we get $C \mathbf{u}=\mathbf{0}$. The reverse inclusion of (3.2) is trivial to be obtained. Thus, from (2.3) we get

$$
\operatorname{dim}[\mathcal{R}(A) \cap \mathcal{N}(A)]=\operatorname{dim} \mathcal{N}(C)=r-\operatorname{rank}(C)=y .
$$

Using again Theorem 2.1, Theorem 3.2 can be restated as follows

Theorem 3.3. For any square complex matrix $A \in \mathbb{C}_{n, n}$, one has

(i) the dimension of $\mathcal{R}(A) \cap \mathcal{R}\left(A^{*}\right)$ is the multiplicity of the singular value 1 in the matrix $P_{\mathcal{R}(A)} P_{\mathcal{R}\left(A^{*}\right)}$.

(ii) if $k$ is the multiplicity of the singular value 0 in the matrix $P_{\mathcal{R}(A)} P_{\mathcal{R}\left(A^{*}\right)}$, then the dimension of $\mathcal{R}(A) \cap \mathcal{R}\left(A^{*}\right)^{\perp}$ is the $\operatorname{rank}(A)+k-n$.

3.2. How far is a matrix from being EP?. The following consequence is a clean measure of the departure of a square matrix from being EP (recall that a square matrix $A$ is said to be EP when $A A^{\dagger}=A^{\dagger} A$ ). More precisely, we have the following result.

Theorem 3.4. Let $A \in \mathbb{C}_{n, n}$. Then

$\left\|A A^{\dagger}-A^{\dagger} A\right\|=\max \left\{\sin \theta: \theta\right.$ is a canonical angle between $\mathcal{R}(A)$ and $\left.\mathcal{R}\left(A^{*}\right)\right\}$.

Proof. Let us represent $A$ as in (2.1). From (2.15) and by following the computations made in (2.23), we have

$$
A A^{\dagger}-A^{\dagger} A=Y\left\{\left[\begin{array}{cc}
I_{r} & 0 \\
0 & 0
\end{array}\right]-\left[\begin{array}{cc}
C^{2} & C S \\
S^{*} C & S^{*} S
\end{array}\right]\right\} Y^{*}=Y\left[\begin{array}{cc}
S S^{*} & -C S \\
-S^{*} C & -S^{*} S
\end{array}\right] Y^{*} .
$$

If we denote $T=Y^{*}\left(A A^{\dagger}-A^{\dagger} A\right) Y$, then $\left\|A A^{\dagger}-A^{\dagger} A\right\|^{2}=\|T\|^{2}=\left\|T T^{*}\right\|$. Thus, to calculate $\left\|A A^{\dagger}-A^{\dagger} A\right\|$, we must first calculate $T T^{*}$. It is straightforward to see that

$$
T T^{*}=\left[\begin{array}{cc}
S S^{*} S S^{*}+C S S^{*} C & -S S^{*} C S+C S S^{*} S \\
-S^{*} C S S^{*}+S^{*} S S^{*} C & S^{*} C^{2} S+S^{*} S S^{*} S
\end{array}\right] .
$$

We shall use (2.15) in order to simplify each block of $T T^{*}$. The upper-left block simplifies to

$$
\begin{aligned}
S S^{*} S S^{*}+C S S^{*} C & =S S^{*}\left(I_{r}-C^{2}\right)+C\left(I_{r}-C^{2}\right) C \\
& =\left(S S^{*}+C^{2}\right)\left(I_{r}-C^{2}\right) \\
& =I_{r}-C^{2} \\
& =S S^{*} .
\end{aligned}
$$


The upper-right block reduces to

$$
-S S^{*} C S+C S S^{*} S=-\left(I_{r}-C^{2}\right) C S+C\left(I_{r}-C^{2}\right) S=0 .
$$

Since $T T^{*}$ is Hermitian and its upper-right block is zero, the lower-left block of $T T^{*}$ is also zero. Now, the lower-right block of $T T^{*}$ simplifies to

$$
S^{*} C^{2} S+S^{*} S S^{*} S=S^{*} C^{2} S+S^{*}\left(I_{r}-C^{2}\right) S=S^{*} S .
$$

Hence, $T T^{*}=S S^{*} \oplus S^{*} S$ and by using that $\left\|A_{1} \oplus A_{2}\right\|=\max \left\{\left\|A_{1}\right\|,\left\|A_{2}\right\|\right\}$ holds for any pair of matrices $A_{1}$ and $A_{2}$ (see, for example, relation 5.2.12 of [18]), we get $\left\|T T^{*}\right\|=\max \left\{\left\|S S^{*}\right\|,\left\|S^{*} S\right\|\right\}=\|S\|^{2}$ and by observing the form of $S$ in Theorem 2.1, we obtain

$$
\|S\|=\max \left\{\sin \theta: \theta \text { is a canonical angle between } \mathcal{R}(A) \text { and } \mathcal{R}\left(A^{*}\right)\right\} .
$$

The following application of Theorem 2.1 is another measure of the departure of a square matrix from being EP. To state this result, let $\mathbb{C}_{n}^{\mathrm{EP}}$ denote the subset of $\mathbb{C}_{n, n}$ composed of EP matrices. Furthermore, if $X \in \mathbb{C}_{n, n}$ and $\mathcal{S} \subset \mathbb{C}_{n, n}$, the expression $\operatorname{dist}(X, \mathcal{S})$ will denote the distance between $X$ and $\mathcal{S}$ (i.e., the infimum of $\|X-Y\|$ when $Y \in \mathcal{S}$ ). The following simple lemma will be useful.

Lemma 3.5. Let $A \in \mathbb{C}_{n, n}$ be represented as in Theorem 2.1. Then $\|A\|=\|M\|$.

Proof. Having in mind the first relation of (2.15), we have $\|A\|^{2}=\left\|A A^{*}\right\|=$ $\left\|M M^{*} \oplus 0\right\|=\left\|M M^{*}\right\|=\|M\|^{2}$.

Theorem 3.6. Let $A \in \mathbb{C}_{n, n}$. Then

$$
\begin{aligned}
& \operatorname{dist}\left(A, \mathbb{C}_{n}^{\mathbb{E P}}\right) \leq \\
& \leq 2\|A\| \sup \left\{\sin (\theta / 2): \theta \text { is a canonical angle between } \mathcal{R}(A) \text { and } \mathcal{R}\left(A^{*}\right)\right\} .
\end{aligned}
$$

Proof. Let us write $A$ as in (2.1) and let $B=Y(M \oplus 0) Y^{*}$. Matrix $B$ is obviously EP because $M$ is nonsingular. From the first relation of (2.15), a simple computation shows $(A-B)(A-B)^{*}=Y\left[\left(2 M\left(I_{r}-C\right) M^{*}\right) \oplus 0\right] Y^{*}$. Therefore,

$$
\operatorname{dist}\left(A, \mathbb{C}_{n}^{\mathrm{EP}}\right)^{2} \leq\|A-B\|^{2}=\left\|2 M\left(I_{r}-C\right) M^{*}\right\| \leq 2\|M\|\left\|I_{r}-C\right\|\left\|M^{*}\right\| .
$$

From Lemma 3.5, one has $\operatorname{dist}\left(A, \mathbb{C}_{n}^{\mathrm{EP}}\right)^{2} \leq 2\|A\|^{2}\left\|I_{r}-C\right\|$. From (2.3) we get

$$
\left\|I_{r}-C\right\|=\sup \left\{1-\cos \theta: \theta \text { is a canonical angle between } \mathcal{R}(A) \text { and } \mathcal{R}\left(A^{*}\right)\right\} .
$$

The use of the trigonometric identity $2 \sin ^{2}(\theta / 2)=1-\cos \theta$ for $\theta \in[0, \pi / 2]$ finishes the proof. $\square$ 
3.3. Characterizations of various classes of matrices. As in [2] we provide characterizations of various known classes of matrices.

TheOREm 3.7. Let $A$ be a square complex matrix represented as in Theorem 2.1. Then

(i) A has group inverse if and only if none of the canonical angles between $\mathcal{R}(A)$ and $\mathcal{R}\left(A^{*}\right)$ is $\pi / 2$, or equivalently, $C$ is nonsingular. In this case, one has

$$
A^{\#}=Y\left[\begin{array}{cc}
C^{-1} M^{-1} & C^{-1} M^{-1} C^{-1} S \\
0 & 0
\end{array}\right] Y^{*} .
$$

(ii) $A$ is a partial isometry (i.e., $A^{*}=A^{\dagger}$ ) if and only if $M$ is unitary.

(iii) $A$ is star-dagger (i.e., $A^{*} A^{\dagger}=A^{\dagger} A^{*}$ ) if and only if $M M^{*} C=C M^{*} M$.

(iv) $A$ is normal (i.e., $A A^{*}=A^{*} A$ ) if and only if all the canonical angles between $\mathcal{R}(A)$ and $\mathcal{R}\left(A^{*}\right)$ are zero and $M$ is normal.

(v) $A$ is an oblique projector (i.e., $A^{2}=A$ ) if and only if $C M=I_{r}$.

(vi) $A$ is an orthogonal projector (i.e., $A^{2}=A=A^{*}$ ) if and only if $M=I_{r}$ and all the canonical angles between $\mathcal{R}(A)$ and $\mathcal{R}\left(A^{*}\right)$ are 0 .

(vii) $A$ is $\operatorname{EP}$ (i.e., $A A^{\dagger}=A^{\dagger} A$, or equivalently, $P_{\mathcal{R}(A)}=P_{\mathcal{R}\left(A^{*}\right)}$ ) if and only if all the canonical angles between $\mathcal{R}(A)$ and $\mathcal{R}\left(A^{*}\right)$ are 0 .

(viii) $A$ is bi-EP (i.e., $A A^{\dagger} A^{\dagger} A=A A^{\dagger} A^{\dagger} A$ or $P_{\mathcal{R}(A)} P_{\mathcal{R}\left(A^{*}\right)}=P_{\mathcal{R}\left(A^{*}\right)} P_{\mathcal{R}(A)}$ ) if and only if all the canonical angles between $\mathcal{R}(A)$ and $\mathcal{R}\left(A^{*}\right)$ are 0 or $\pi / 2$.

(ix) $A$ is a contraction (i.e., $\|A\| \leq 1$ ) if and only if $M$ is a contraction.

Proof. Let us write $A$ as in (2.1).

(i) One has that $A$ has group inverse if and only if $\operatorname{rank}\left(A^{2}\right)=\operatorname{rank}(A)[4$, Sec. 4.4]. Moreover, as is easy to see from (2.2) we have that none of the canonical angles between $\mathcal{R}(A)$ and $\mathcal{R}\left(A^{*}\right)$ is $\pi / 2$ if and only if $C$ is nonsingular. Furthermore, we have

$$
A^{2}=Y\left(M C \oplus I_{n-r}\right) Y^{*} A
$$

Assume that $C$ is nonsingular. From (3.4) we get $\operatorname{rank}\left(A^{2}\right)=\operatorname{rank}(A)$, because premultiplying by nonsingular matrices does not change the rank.

Assume that $C$ is singular. Since $M^{*}$ is nonsingular, there exists $\mathbf{u} \in \mathbb{C}_{r, 1}$ such that $\mathbf{u} \neq \mathbf{0}$ and $C M^{*} \mathbf{u}=\mathbf{0}$. Define

$$
\mathbf{v}=Y\left[\begin{array}{l}
\mathbf{u} \\
\mathbf{0}
\end{array}\right] \in \mathbb{C}_{n, 1}
$$

Since $\mathbf{u} \neq \mathbf{0}$ and $Y$ is nonsingular ( $Y$ is unitary) we have $\mathbf{v} \neq \mathbf{0}$. Furthermore, from 
(3.4) we get

$$
\left(A^{*}\right)^{2} \mathbf{v}=A^{*} Y\left[\begin{array}{cc}
C M^{*} & 0 \\
0 & I_{n-r}
\end{array}\right] Y^{*} Y\left[\begin{array}{l}
\mathbf{u} \\
\mathbf{0}
\end{array}\right]=\mathbf{0}
$$

and

$$
A^{*} \mathbf{v}=Y\left[\begin{array}{cc}
C M^{*} & 0 \\
S^{*} M^{*} & 0
\end{array}\right] Y^{*} Y\left[\begin{array}{l}
\mathbf{u} \\
\mathbf{0}
\end{array}\right]=Y\left[\begin{array}{c}
\mathbf{0} \\
S^{*} M^{*} \mathbf{u}
\end{array}\right] .
$$

If $A^{*} \mathbf{v}=\mathbf{0}$, then $S^{*} M^{*} \mathbf{u}=\mathbf{0}$. Recall that by the choice of $\mathbf{u}$ we have $C M^{*} \mathbf{u}=\mathbf{0}$, and now, from Lemma 3.1 we get $M^{*} \mathbf{u}=\mathbf{0}$, which is a contradiction with $\mathbf{u} \neq \mathbf{0}$ and the nonsingularity of $M^{*}$. Hence $\mathbf{v} \in \mathcal{N}\left(\left(A^{*}\right)^{2}\right)$ and $\mathbf{v} \notin \mathcal{N}\left(A^{*}\right)$, therefore the null spaces of $A^{*}$ and $\left(A^{*}\right)^{2}$ are not equal, and thus, the ranks of $A$ and $A^{2}$ are not equal.

To finish the proof of this item, let us observe that the expression (3.3) can be verified by direct verifications.

(ii) Using the expressions (2.1) and (2.22) we have $A^{*}=A^{\dagger}$ if and only if $C M^{*}=$ $C M^{-1}$ and $S^{*} M^{*}=S^{*} M^{-1}$. Lemma 3.1 leads to

$$
C M^{*}=C M^{-1} \text { and } S^{*} M^{*}=S^{*} M^{-1} \quad \Longleftrightarrow \quad M^{-1}=M^{*} .
$$

(iii) The proof is similar as in (ii).

(iv) We have $A A^{*}=A^{*} A$ if and only if the following four equalities are satisfied

$$
M M^{*}=C M^{*} M C, \quad 0=C M^{*} M S, \quad 0=S^{*} M^{*} M C, \quad 0=S^{*} M^{*} M S .
$$

In view of the nonsingularity of $M$ we have $0=S^{*} M^{*} M S \Leftrightarrow 0=(M S)^{*}(M S) \Leftrightarrow$ $M S=0 \Leftrightarrow S=0$. From the first relation of (2.15) we have $S=0 \Leftrightarrow C^{2}=I_{r}$. Having in mind that $C$ is a diagonal matrix with nonnegative real numbers on its diagonal, we have $C^{2}=I_{r} \Leftrightarrow C=I_{r}$. Therefore, $A A^{*}=A^{*} A$ if and only if $C=I_{r}$ and $M M^{*}=M^{*} M$. From (2.3), the equality $C=I_{r}$ is equivalent to saying that all the canonical angles between $\mathcal{R}(A)$ and $\mathcal{R}\left(A^{*}\right)$ are zero.

(v) We have $A^{2}=A$ if and only if $M C M C=M C$ and $M C M S=M S$. Taking into account that $M$ is nonsingular we get $A^{2}=A$ if and only if $C M C=C$ and $C M S=S$. Lemma 3.1 finishes the proof of this item.

(vi) Since $M$ is nonsingular, we have $A^{*}=A$ if and only if $M C=C M^{*}$ and $S=0$. In view of (2.15) and item (v) we get $A^{2}=A=A^{*}$ if and only if $C=M=I_{r}$.

(vii) It follows from Theorem 3.4.

(viii) It is easily seen that

$$
A A^{\dagger} A^{\dagger} A-A^{\dagger} A A A^{\dagger}=Y\left[\begin{array}{cc}
0 & C S \\
-S^{*} C & 0
\end{array}\right] Y^{*} .
$$


Thus, $A$ is bi-EP if and only if $C S=0$. If $C S=0$, from the first equality of (2.15) we get $C^{3}=C$. From (2.3), (2.4), and (2.14) we have

$$
p=0, \quad C=0_{y, y} \oplus I_{x}, \quad S=I_{y} \oplus 0_{x, t} .
$$

Obviously, (3.5) implies $C S=0$. Evidently, from Theorem 2.1, the conditions given in (3.5) are equivalent to saying that all the canonical angles between $\mathcal{R}(A)$ and $\mathcal{R}\left(A^{*}\right)$ are 0 or $\pi / 2$.

(ix) It is trivial from Lemma 3.5.

3.4. Applications to some partial matrix orderings. In the following, three partial orderings in $\mathbb{C}_{n, n}$ will be studied. The first of them is the star ordering introduced in [10], which is defined by

$$
A \stackrel{*}{\leq} B \quad \Longleftrightarrow \quad A^{\dagger} A=A^{\dagger} B \text { and } A A^{\dagger}=B A^{\dagger},
$$

or alternatively,

$$
A \stackrel{*}{\leq} B \quad \Longleftrightarrow \quad A^{*} A=A^{*} B \text { and } A A^{*}=B A^{*} .
$$

The second one is the sharp ordering defined in [19]:

$$
A \stackrel{\#}{\leq} B \quad \Longleftrightarrow \quad A^{\#} A=A^{\#} B \text { and } A A^{\#}=B A^{\#},
$$

when $A$ and $B$ have group inverse. It is easy to verify that

$$
A \stackrel{\#}{\leq} B \quad \Longleftrightarrow \quad A B=A^{2}=B A .
$$

Furthermore, we will consider the minus ordering defined in [13]. An equivalent form of this ordering is the following $[7,17]$ :

$$
A \overline{\leq} B \quad \Longleftrightarrow \quad A B^{\dagger} B=A, \quad B B^{\dagger} A=A, \quad A B^{\dagger} A=A
$$

In [3], the authors provide handy tools to verify whether given matrices $A$ and $B$ satisfy $A \stackrel{*}{\leq} B, A \stackrel{\#}{\leq} B$, or $A \stackrel{-}{\leq} B$ when $A$ is written as in (1.1). However, the characterizations given in [3] of $B \stackrel{*}{\leq} A, B \stackrel{\#}{\leq} A$, and $B \overline{\leq} A$, when $A$ is written as in (1.1), lead to various sets of matrix equations very difficult to handle. These sets of equations, under particular situations, can be reduced, as the authors of [3] showed. In the forthcoming Theorem 3.9 we find a more general situation where the characterizations of $B \stackrel{*}{\leq} A, B \stackrel{\#}{\leq} A$, and $B \underset{\leq}{\leq} A$, when $A$ is written as in (2.1) are easy to check. 
Before doing this, the following result, given by Cao in [6], will be helpful.

Theorem 3.8. If $A \in \mathbb{C}_{r, r}, B \in \mathbb{C}_{r, s}, C \in \mathbb{C}_{s, s}$, and $M=\left[\begin{array}{cc}A & B \\ 0 & C\end{array}\right]$, then there exists $M^{\#}$ if and only if there exist $A^{\#}$ and $C^{\#}$, and in addition $\left(I_{r}-A A^{\#}\right) B\left(I_{s}-\right.$ $\left.C C^{\#}\right)=0$ holds. Furthermore, when $M^{\#}$ exists, it is given by

$$
M^{\#}=\left[\begin{array}{cc}
A^{\#} & \left(A^{\#}\right)^{2} B\left(I_{s}-C C^{\#}\right)+\left(I_{r}-A A^{\#}\right) B\left(C^{\#}\right)^{2}-A^{\#} B C^{\#} \\
0 & C^{\#}
\end{array}\right] .
$$

Theorem 3.9. Let $A, B \in \mathbb{C}_{n, n}$ and let $A$ be of the form (2.1). Assume that $A$ has group inverse.

(i) $B \stackrel{*}{\leq} A$ if and only if $B$ can be written as

$$
B=Y\left[\begin{array}{cc}
B_{1} & B_{2} \\
0 & 0
\end{array}\right] Y^{*}, \quad B_{1} \in \mathbb{C}_{r, r}, \quad B_{2} \in \mathbb{C}_{n-r, r},
$$

where $B_{1}$ and $B_{2}$ satisfy

$$
(3.10) B_{1}^{*} B_{1}=B_{1}^{*} M C, \quad B_{2}=B_{1} C^{-1} S, \quad B_{1} C^{-2} B_{1}^{*}=M C^{-1} B_{1}^{*}
$$

(ii) If $B$ has group inverse, then $B \stackrel{\#}{\leq} A$ if and only if $B$ can be written as in (3.9) and $B_{1}, B_{2}$ satisfy

$$
B_{1} \stackrel{\#}{\leq} M C \quad \text { and } \quad B_{2}=C^{-1} M^{-1} B_{1} M S .
$$

(iii) $B \overline{\leq} A$ if and only if $B$ can be written as in (3.9) and $B_{1}, B_{2}$ satisfy

$$
B_{1} C^{-1} M^{-1} B_{1}=B_{1} \quad \text { and } \quad B_{1} C^{-1} M^{-1} B_{2}=B_{2} .
$$

Proof. Let us write $A$ as in (2.1). Since $A$ has group inverse, by Theorem 3.7, $C$ is nonsingular. Also, let us write $B$ as

$$
B=Y\left[\begin{array}{ll}
B_{1} & B_{2} \\
B_{3} & B_{4}
\end{array}\right] Y^{*}, \quad B_{1} \in \mathbb{C}_{r, r}, \quad B_{4} \in \mathbb{C}_{n-r, n-r} .
$$

(i) We obtain from (2.1) and (3.13)

$$
\begin{aligned}
& B^{*} B=Y\left[\begin{array}{cc}
B_{1}^{*} B_{1}+B_{3}^{*} B_{3} & B_{1}^{*} B_{2}+B_{3}^{*} B_{4} \\
B_{2}^{*} B_{1}+B_{4}^{*} B_{3} & B_{2}^{*} B_{2}+B_{4}^{*} B_{4}
\end{array}\right] Y^{*}, \\
& B B^{*}=Y\left[\begin{array}{ll}
B_{1} B_{1}^{*}+B_{2} B_{2}^{*} & B_{1} B_{3}^{*}+B_{2} B_{4}^{*} \\
B_{3} B_{1}^{*}+B_{4} B_{2}^{*} & B_{3} B_{3}^{*}+B_{4} B_{4}^{*}
\end{array}\right] Y^{*},
\end{aligned}
$$




$$
B^{*} A=Y\left[\begin{array}{ll}
B_{1}^{*} M C & B_{1}^{*} M S \\
B_{2}^{*} M C & B_{2}^{*} M S
\end{array}\right] Y^{*}
$$

and

$$
A B^{*}=Y\left[\begin{array}{cc}
M C B_{1}^{*}+M S B_{2}^{*} & M C B_{3}^{*}+M S B_{4}^{*} \\
0 & 0
\end{array}\right] Y^{*} .
$$

Assume $B \stackrel{*}{\leq} A$. In particular we have $B B^{*}=A B^{*}$, which in view of the (3.14), (3.15), (3.16), and (3.17), leads to $B_{3} B_{3}^{*}+B_{4} B_{4}^{*}=0$, i.e., $B_{3}=0$ and $B_{4}=0$.

Taking these relations into account, it is seen that $B \stackrel{*}{\leq} A$ implies

$$
B_{1}^{*} B_{1}=B_{1}^{*} M C, \quad B_{1}^{*} B_{2}=B_{1}^{*} M S, \quad B_{2}^{*} B_{1}=B_{2}^{*} M C, \quad B_{2}^{*} B_{2}=B_{2}^{*} M S
$$

and

$$
B_{1} B_{1}^{*}+B_{2} B_{2}^{*}=M C B_{1}^{*}+M S B_{2}^{*} .
$$

The first equality of (3.18) implies that $B_{1}^{*} M C$ is Hermitian, thus

$$
M^{-*} C^{-1} B_{1}^{*}=B_{1} C^{-1} M^{-1} .
$$

From the second and third relations of (3.18) we obtain

$$
B_{1}^{*} M S=B_{1}^{*} B_{2}=\left(B_{2}^{*} B_{1}\right)^{*}=\left(B_{2}^{*} M C\right)^{*}=C M^{*} B_{2} .
$$

Solving $B_{2}$ and using (3.20) we get

$$
B_{2}=M^{-*} C^{-1} B_{1}^{*} M S=B_{1} C^{-1} M^{-1} M S=B_{1} C^{-1} S .
$$

Now, we shall simplify each side of (3.19) by using (2.15):

$$
\begin{aligned}
B_{1} B_{1}^{*}+B_{2} B_{2}^{*} & =B_{1} B_{1}^{*}+B_{1} C^{-1} S S^{*} C^{-1} B_{1}^{*} \\
& =B_{1} B_{1}^{*}+B_{1}\left(C^{-1}\left(I_{r}-C^{2}\right) C^{-1}\right) B_{1}^{*} \\
& =B_{1} C^{-2} B_{1}^{*}
\end{aligned}
$$

and

$$
\begin{aligned}
M C B_{1}^{*}+M S B_{2}^{*} & =M C B_{1}^{*}+M S S^{*} C^{-1} B_{1}^{*} \\
& =M\left(C+\left(I_{r}-C^{2}\right) C^{-1}\right) B_{1}^{*} \\
& =M C^{-1} B_{1}^{*} .
\end{aligned}
$$

Hence the necessity is proved. 
Now, we will prove that if $B$ is written as in (3.9) and the conditions (3.10) are satisfied, then $B \stackrel{*}{\leq} A$. In other words, we will verify (3.18) and (3.19). By the computations made in (3.21) and (3.22), condition (3.19) holds. Thus, it only remains to prove the second, third, and fourth relations of (3.18):

$$
\begin{gathered}
B_{1}^{*} B_{2}=B_{1}^{*} B_{1} C^{-1} S=B_{1}^{*} M C C^{-1} S=B_{1}^{*} M S, \\
B_{2}^{*} B_{1}=S^{*} C^{-1} B_{1}^{*} B_{1}=S^{*} C^{-1} B_{1}^{*} M C=B_{2}^{*} M C,
\end{gathered}
$$

and

$$
B_{2}^{*} B_{2}=S^{*} C^{-1} B_{1}^{*} B_{1} C^{-1} S=S^{*} C^{-1} B_{1}^{*} M C C^{-1} S=B_{2}^{*} M S .
$$

The sufficiency is proved.

(ii) From (2.1) and (3.13) it follows

$$
\begin{gathered}
A B=Y\left[\begin{array}{cc}
M C B_{1}+M S B_{3} & M C B_{2}+M S B_{4} \\
0 & 0
\end{array}\right] Y^{*} \\
B A=Y\left[\begin{array}{cc}
B_{1} M C & B_{1} M S \\
B_{3} M C & B_{3} M S
\end{array}\right] Y^{*}
\end{gathered}
$$

and

$$
B^{2}=Y\left[\begin{array}{cc}
B_{1}^{2}+B_{2} B_{3} & B_{1} B_{2}+B_{2} B_{4} \\
B_{3} B_{1}+B_{4} B_{3} & B_{3} B_{2}+B_{4}^{2}
\end{array}\right] Y^{*}
$$

Assume that $B \stackrel{\#}{\leq} A$, i.e., $A B=B A=B^{2}$ holds. From $A B=B A$ we get $M C B_{1}+M S B_{3}=B_{1} M C, \quad M C B_{2}+M S B_{4}=B_{1} M S, \quad B_{3} M C=0, \quad B_{3} M S=0$. The nonsingularity of $M$ and $C$ leads to $B_{3}=0$. Therefore, we obtain

$$
M C B_{1}=B_{1} M C, \quad M C B_{2}+M S B_{4}=B_{1} M S, \quad B_{3}=0 .
$$

Taking into account that $B_{3}=0$, from $B A=B^{2}$ we get

$$
B_{1} M C=B_{1}^{2}, \quad B_{1} M S=B_{1} B_{2}+B_{2} B_{4}, \quad 0=B_{4}^{2} .
$$

Since $B=Y\left[\begin{array}{cc}B_{1} & B_{2} \\ 0 & B_{4}\end{array}\right] Y^{*}$, we can apply Theorem 3.8 obtaining that $B_{4}$ has group inverse. Since $0=B_{4}^{2}$, premultiplying by $B_{4}^{\#}$ we get $B_{4}=0$. Hence (3.23) and (3.24) reduce to

$$
B_{1}^{2}=M C B_{1}=B_{1} M C, \quad B_{1} B_{2}=M C B_{2}=B_{1} M S, \quad B_{3}=0, \quad B_{4}=0 .
$$


In particular, from (3.25), we get $B_{1} \stackrel{\#}{\leq} M C$ and $B_{2}=C^{-1} M^{-1} B_{1} M S$.

Let us prove that if $B$ is written as in (3.9) and $B_{1}, B_{2}$ satisfy (3.11), then $B \stackrel{\#}{\leq} A$, or in other words, let us prove $A B=B A=B^{2}$. If we write $A$ as in (2.1) and $B$ as in (3.9), we obtain $A B=B A=B^{2}$ if and only if $B_{1} M C=M C B_{1}=B_{1}^{2}$ and $B_{1} M S=M C B_{2}=B_{1} B_{2}$. In view of the assumptions, it only remains to prove $B_{1} B_{2}=B_{1} M S$ :

$$
B_{1} B_{2}=B_{1} C^{-1} M^{-1} B_{1} M S=C^{-1} M^{-1} B_{1}^{2} M S=C^{-1} M^{-1} M C B_{1} M S=B_{1} M S .
$$

(iii) Since $B \overline{\leq} A$, the corresponding version of (3.8) leads to $A A^{\dagger} B B=B$, which yields $B_{3}=0$ and $B_{4}=0$. From $B A^{\dagger} A=B$ we get $B_{1} C^{2}+B_{2} S^{*} C=B_{1}$ and $B_{1} C S+B_{2} S^{*} S=B_{2}$. Taking into account (2.15), these relations yield

$$
B_{2} S^{*} C=B_{1} S S^{*}, \quad B_{2}\left(C \oplus I_{t}\right)^{2}=B_{1} S\left(C \oplus I_{t}\right) .
$$

Since $C$ is nonsingular, the second relation of (3.26) yields $B_{2}\left(C \oplus I_{t}\right)=B_{1} S$. Parenthetically, let us remark that postmultiplying $B_{2}\left(C \oplus I_{t}\right)=B_{1} S$ by $S^{*}$, and using the third relation of (2.15) yields the first relation of (3.26). Therefore, the two relations of (3.26) are equivalent to the simpler relation

$$
B_{2}\left(C \oplus I_{t}\right)=B_{1} S
$$

It further follows that $B A^{\dagger} B=B$, obtained from the third condition of (3.8), is equivalent to

$$
\left(B_{1} C+B_{2} S^{*}\right) M^{-1} B_{1}=B_{1}, \quad\left(B_{1} C+B_{2} S^{*}\right) M^{-1} B_{2}=B_{2} .
$$

Now let us simplify $B_{1} C+B_{2} S^{*}$. To do this, we use (2.15) and (3.27). Observe that the third relation of $(2.15)$ can be equivalently written as $S\left(\widehat{C} \oplus I_{t}\right)^{-1}=C^{-1} S$, and (3.27) is equivalent to $B_{2}=B_{1} S\left(C \oplus I_{t}\right)^{-1}$. Therefore,

$$
\begin{aligned}
B_{1} C+B_{2} S^{*} & =B_{1}\left[C+S\left(C \oplus I_{t}\right)^{-1} S^{*}\right] \\
& =B_{1}\left(C+C^{-1} S S^{*}\right) \\
& =B_{1} C^{-1}\left(C^{2}+S S^{*}\right) \\
& =B_{1} C^{-1} .
\end{aligned}
$$

And thus, (3.28) is equivalent to (3.12).

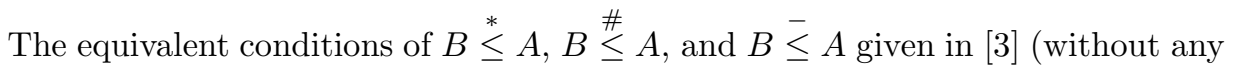
assumption on matrix $B$ ) are rather difficult to handle. In [3], the authors simplified the aforementioned conditions under the assumption that $A$ is idempotent or EP. Let 
us observe that conditions (3.10), (3.11), and (3.12) are much easier to manage, and let us remark that these conditions were obtained under the assumption that $A$ has group inverse, a condition on $A$ much more general that being idempotent or EP.

\section{REFERENCES}

[1] O.M. Baksalary and J. Benítez. On linear combinations of two commuting hypergeneralized projectors. Computers \&5 Mathematics with Applications, 56:2481-2489, 2008.

[2] O.M. Baksalary and G. Trenkler. Characterizations of EP, normal, Hermitian matrices. Linear and Multilinear Algebra, 56:299-304, 2008.

[3] O.M. Baksalary, G. Trenkler, and G.P.H. Styan. On a matrix decomposition of Hartwig and Spindelböck. Linear Algebra and its Applications, 430:2798-2812, 2009.

[4] A. Ben-Israel and T.N.E. Greville. Generalized Inverses. Theory and Applications (2nd edition), Springer-Verlag, 2002.

[5] J. Benítez and V. Rakočević. Applications of CS decomposition in linear combinations of two orthogonal projectors. Applied Mathematics and Computation, 203:761-769, 2008.

[6] Cao CG. Some results of group inverses for partitioned matrices over skew fields, Journal of Natural Science of Heilongjiang University, 18(3):5-7, 2001.

[7] R.E. Cline and R.E. Funderlic. The rank of a difference of matrices and associated generalized inverses. Linear Algebra and its Applications, 24:185-215, 1979.

[8] J. Dauxois and G.M. Nkiet. Canonical analysis of two Euclidean subspaces and its applications. Linear Algebra and its Applications, 264:355-388, 1997.

[9] C. Davis and W.M. Kahan. The rotation of eigenvectors by a perturbation III. SIAM Journal on Numerical Analysis, 7:1-46, 1970.

[10] M.P. Drazin. Natural structures on semigroups with involution. Bulletin of the American Mathematical Society, 84:139-141, 1978.

[11] A. Galantai and Cs.J Hegedus. Jordan's principal angles in complex vector spaces Numerical Linear Algebra with Applications, 13:589-598, 2006.

[12] G.H. Golub and C.F. van Loan. Matrix Computations (3rd edition), Johns Hopkins Studies in the Mathematical Sciences, Johns Hopkins University Press, Baltimore, MD, 1996.

[13] R.E. Hartwig. How to partially order regular elements. Mathematica Japonica, 25:1-13, 1980.

[14] R.E. Hartwig and K. Spindelböck. Matrices for which $A^{*}$ and $A^{\dagger}$ commute. Linear and Multilinear Algebra, 14:241-256, 1984.

[15] H. Hotelling. Relation between two sets of variables. Biometrika, 28:322-377, 1936.

[16] E.R. Jessup, M.W. Berry and Z. Drmac. Matrices, vector spaces, and information retrieval. SIAM Review, 41:335-362, 1999.

[17] G. Marsaglia and G.P.H. Styan. Equalities and inequalities for ranks of matrices. Linear and Multilinear Algebra, 2:269-292, 1974.

[18] C.D. Meyer. Matrix Analysis and Applied Linear Algebra. SIAM, Philadelphia, PA, 2000.

[19] S.K. Mitra. On group inverses and the sharp order. Linear Algebra and its Applications, 92:1737, 1987.

[20] C.C. Paige and M. Wei. History and generality of the CS decomposition. Linear Algebra and its Applications, 208/209:303-326, 1994.

[21] V. Rakočević and H.K. Wimmer. A variational characterization of canonical angles between subspaces. Journal of Geometry, 78:122-124, 2003.

[22] I.B. Risteski and K.G. Trencevski. Principal Values and Principal Subspaces of Two Subspaces of Vector Spaces with Inner Product. Beiträge zur Algebra und Geometrie, 42:289-300, 2001.

[23] G.W. Stewart. Matrix Algorithms, Volume II: Eigensystems. SIAM. Philadelphia, PA 2001. 
Electronic Journal of Linear Algebra ISSN 1081-3810

A publication of the International Linear Algebra Society

ELA

[24] G. Trenkler. On oblique and orthogonal projectors. In Contributions to Probability and Statistics: Applications and Challenges, Proceedings of the International Statistics Workshop World Scientific, Singapore, 178-191, 2006.

[25] H.K. Wimmer. Canonical angles of unitary spaces and perturbations of direct complements. Linear Algebra and its Applications, 287:373-379, 1999. 